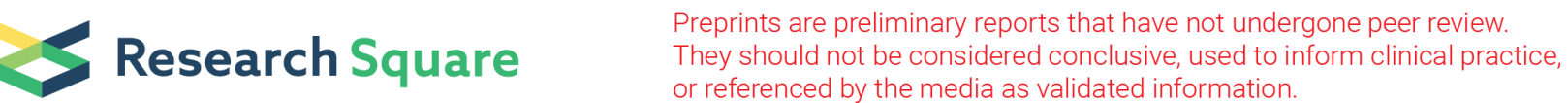

\section{Effects of Prebiotic Supplement on Gut Microbiota, Drug Bioavailability and Adverse Effects in Patients with Colorectal Cancer at Different Primary Tumor Locations Receiving Chemotherapy: Study Protocol for a Randomized Clinical Trial}

Yanmin Li

Jiangnan University

Hong Cao

Affiliated Hospital of Jiangnan University

Bojian Fei

Affiliated Hospital of Jiangnan University

Chuanqing Bao

Affiliated Hospital of Jiangnan University

Jianmin Xu

Affiliated Hospital of Jiangnan University

Huaxiang Cao

Affiliated Hospital of Jiangnan University

Yong Mao

Affiliated Hospital of Jiangnan University

Xiaoping Chen

Affiliated Hospital of Jiangnan University

\section{Xiang Gao}

Affiliated Hospital of Jiangnan University

Wei Zhao ( $\nabla$ zhaow@jiangnan.edu.cn )

Jiangnan University https://orcid.org/0000-0001-9495-8508

Feng Zhang

Affiliated Hospital of Jiangnan University

\section{Research Article}

Keywords: Colorectal cancer, Primary tumor locations, Gut microbiota, Adverse effects, Drug bioavailability 
Posted Date: January 11th, 2022

DOl: https://doi.org/10.21203/rs.3.rs-907838/v1

License: (c) (i) This work is licensed under a Creative Commons Attribution 4.0 International License. Read Full License 


\section{Abstract}

Background: The prevalence of colorectal cancer (CRC) worldwide is a huge challenge to human health. Primary tumor locations found to impact prognosis

and response to therapy. The important role of gut microbiota in the progression and treatment of CRC has led to many attempts of alleviating chemotherapy-induced adverse effects using microecologics. However, the underlying mechanism of the difference in the prognosis of different primary tumor locations and the synergistic effect of prebiotics on chemotherapy need to be further elucidated. This study aims to explore the differences in tumor microbiota and examine the effectiveness of xylooligosaccharides (XOS) on gut microbiota, adverse effects, and bioavailability of chemotherapy drugs in CRC patients at different primary tumor locations.

Methods: This is a double-blinded, randomized, parallel controlled clinical trial. Participants with leftsided CRC ( $L S C R C, n=50)$ and right-sided CC (RSCC, $n=50)$ will randomly allocated to prebiotic group ( $n$ $=25)$ or control group $(n=25)$ and will receive either a daily XOS $(3 \mathrm{~g} / \mathrm{d})$ or placebo, respectively, for 12 weeks. The primary outcomes will be the differences in the mucosa microbiota composition at different tumor locations, and differences in gut microbiota composition, adverse effects, and blood concentration of capecitabine posttreatment. The secondary outcomes will include other blood indicators, short-chain fatty acids (SCFAs) concentration, quality of life, and mental health.

Discussion: This study will reveal the potential benefits of prebiotic for improving the gut microbiota composition, alleviating the adverse effects, and improving the efficacy of chemotherapy in patients with CRC. In addition, this study will provide data on the different distribution of tumor microbiota and the different changes of gut microbiota during treatment in LSCRC and RSCC, which may provide novel insights into personalized cancer treatment strategies based on primary tumor locations and gut microbiota in the future.

Trial registration: Chinese Clinical Trial Registry(www.chictr.org.cn): ChiCTR2100046237. Registered on 12 May 2021.

\section{Introduction}

Worldwide, colorectal cancer (CRC) has become the third-ranked cancer in incidence and the secondranked in mortality. It is estimated that 935,000 people died of CRC in 2020 according to the 2020 Global Cancer Statistics [1]. Lacking of physical activity, obesity, and diet were all independent factors related to the risk of CRC [2]. Some studies suggest that factors causing the imbalance of intestinal flora may also be significant to the carcinogenesis of CRC $[3,4]$. Based on location, CRC can be classified into left-sided CRC (LSCRC) and right-sided CC(RSCC). RSCC is defined as any tumor occurring in the cecum, ascending colon, hepatic flexure, or first two-thirds of the transverse colon, while the LSCRC is defined as any tumor occurring in the latter one-third of the transverse colon, splenic flexure, descending colon, sigmoid colon and rectum [5]. Different primary tumor locations differ in incidence, pathogenesis, clinical 
characteristics, survival prognosis, molecular biological characteristics, and gut microbiota [6]. Several studies have demonstrated that patients with LSCRC had a significantly better prognosis compared with those with RSCC [7-11].

Increasing evidence supports the character of intestinal flora in the pathogenesis and treatment of CRC. Fusobacterium nucleatum adheres, invades, and induces carcinogenic and inflammatory responses via its unique FadA adhesion to activate the growth of CRC cells [12]. Other than the direct effect of specific bacteria on partial tissues, the broader bacterial community may regulate the risk and progression of CRC via competitive rejection and other mechanisms. Gut microbiota-dependent short-chain fatty acids (SCFAs) generation fermented from dietary fiber exert protection against colorectal tumorigenesis in rodents [13].

At present, cytotoxic drugs are generally applied for postoperative adjuvant therapy or advanced CRC patients, usually accompanied by a series of adverse effects (such as constipation, diarrhea, nausea, and bone marrow suppression) and individual differences in drug efficacy $[14,15]$. In recent years, with the rise of 'pharmacomicrobiomics', the significance of the intestinal flora in regulating the host response to chemotherapeutic drugs is increasingly recognized [16]. Intestinal flora can modulate the anti-tumor activity of chemotherapy drugs [17]. The killing effectiveness of 5-Fu on CRC can be enhanced by bacterial-derived urolithin A [18]. Importantly, proteobacteria can promote the deglycosylation of capecitabine [19]. Moreover, some studies have reported that probiotics/prebiotics can reduce the adverse effects of chemotherapy. Lactobacillus casei variety rhamnosus plays a preventive role in FOLFOXrelated intestinal mucositis in CRC-bearing mice [20]. In CRC patients, L. rhamnosus GG consumption reduced the incidence of 5-FU-induced severe diarrhea and abdominal discomfort compared with guar gum fiber [21]. Lu et al. found that synbiotics (containing a variety of probiotics and prebiotic ingredients) can help relieve postoperative chemotherapy-related adverse effects, further improving the quality of life (QoL) in CRC patients [22]. Hence, targeting the gut microbiota in clinical practice to modulate the efficacy and toxicity of chemotherapeutic drugs has a promising future.

Although there have been some reports on the effects of microecologics on modulating intestinal flora and reducing adverse effects in patients with CRC during chemotherapy, they are still very limited. Through our current knowledge, using microecologics to interfere with the bioavailability of chemotherapeutic agents in a microbiota-dependent manner has not been reported, as well as differences in the intervention effect of prebiotics on the intestinal flora of CRC patients at different primary tumor locations.

Xylooligosaccharide (XOS) is a mixture of oligosaccharides consist of 2-9 xylose units connected by $\beta-1,4$-glycosidic bonds $[23,24]$, which can scape digestion by host enzymes in the small intestine and enter the large intestine directly, and served as major substrates for gut bacterial growth. XOS can be utilized by beneficial bacteria first in the intestine, enriching bifidobacteria and lactobacillus, and inhibiting the proliferation of harmful bacteria such as $E$. coli and Salmonella enteritidis to reduce the production of harmful substances [25-27]. The proliferation effect of XOS on bifidobacteria is about 20 
times that of other functional oligosaccharides, with higher selectivity $[28,29]$. XOS can facilitate the generation of SCFAs such as acetic acid, propionic acid, and butyric acid [30], as well as other organic acids such as lactic acid, succinate, formic acid, isobutyric acid, and isohexanoic acid [31], which play important roles in preventing various intestinal diseases. XOS was found to increase the moisture content of stool and improve constipation [32]. In addition, XOS have a strong ability to adsorb pathogens, thereby preventing diarrhea [33]. Evidence from in vitro and animal research indicates that XOS play roles in inhibiting the secretion of inflammatory cytokines and have an effect on anti-inflammatory and antitumor $[34,35]$. Chronic toxicology studies on XOS show that it is safe and reliable with no toxicity in humans, dogs, rodents, and other animals [36-38].

This study will explore the difference of tumor microbiota and the different gut microbiota changes during treatment between LSCRC and RSCC. This will give us a better understanding of the tumor microenvironment, a better understanding of the mechanism of cancer occurrence and progression, and provide a basis for new precision treatment and diagnosis methods in the future. Prebiotics are potentially innovative, inexpensive, and easy-to-persist interventions which can be used for the conservative management of CRC patients. This trial aims to explore the effect of XOS on increasing the bioavailability of cytotoxic drug, reducing the adverse effects of chemotherapy, and improving the QoL of CRC patients by modifying gut microbiota.

\section{Methods And Analysis \\ Study design}

This study is a single-center, randomized, parallel controlled clinical trial conducted at the Affiliated Hospital of Jiangnan University in Wuxi, China. Participants with LSCRC and RSCC will receive prebiotic or placebo intervention during postoperative chemotherapy for three months. The study flow is shown in Fig. 1. Study procedures and data collection are shown in Fig. 2 based on SPIRIT (Standard Protocol Items: Recommendations for Interventional Trials) [39].

\section{Inclusion criteria}

1. Patients diagnosed with primary CRC who will be treated with XELOX chemotherapy;

2. Patients aged $18-80$ years;

3. Patients with an Eastern Cooperative Oncology Group (ECOG) performance status score of 0 or 1 , absolute number of neutrophils $\geq 1.5 \times 10^{9} / \mathrm{L}$, platelets $\geq 100 \times 10^{9} / \mathrm{L}$, serum creatinine $\leq 1.5$ times upper limit of normal (ULN), total bilirubin $\leq 1.5$ times ULN, aspartate transaminase/alaninetransaminase $\leq 2.5$ times ULN and carcinoembryonic antigen within the normal range;

4. Patients have not received preoperative neoadjuvant radiotherapy and chemical treatment;

5. Written informed consent. 


\section{Non-inclusion criteria}

1. Suffer from other tumors at the same time;

2. Patient has other digestive tract diseases except gastrointestinal tumors, such as inflammatory bowel disease, acute gastroenteritis, etc.;

3. Those who have taken antibiotics, drugs or food containing probiotics within 2 weeks;

4. Those with active infections or those with mental illness, cardiovascular and cerebrovascular diseases, severe cardiopulmonary dysfunction, and other serious diseases that are not suitable for chemotherapy;

5. Those who have undergone multiple courses of chemotherapy, extensive radiotherapy, advanced age, bone marrow metastasis, severe infection, adrenal insufficiency, and severe illness;

6. Women during pregnancy;

7. Patients who cannot receive treatment on time and cannot cooperate fully.

\section{Exclusion criteria}

Patients who change medication regimen in the chemotherapy cycle, patients who receive antibiotics or other probiotics/ prebiotics during the intervention, individuals who do not take the prebiotics consistently, and individuals who suffer complications that may be associated with prebiotics will be excluded.

\section{Randomization and blinding}

After signing the informed consent form and complete baseline questionnaires, patients with LSCRC ( $\mathrm{n}=$ $50)$ and RSCC $(n=50)$ will be randomly divided into prebiotic group $(n=25)$ and control group $(n=25)$ in a 1:1 allocation ratio, respectively. The random number will be generated by a computer and sealed in envelopes by an investigator who will not be involved in running the study, and placed in a safe place. Once a participant meets the enrollment conditions and signs the informed consent can open one envelope in the order of enrollment time. Prebiotic and placebo will be packaged in opaque bags without any graphics, and their appearance and smell are the same. A third party who has no direct involvement will stick the "A" and "B" codes on the packaging boxes. Before data analysis, participants and investigators will not know the contents of the bags, allocation, and study treatments.

\section{Setting and interventions}

Posters and leaflets will be accessible in the outpatient and inpatient departments of the hospital so that interested candidates with $\mathrm{CRC}$ can get in touch with investigators to participate in the screening process. The eligible participants will be invited for the first visit, and two trained investigators will explain the 
study procedures in detail to them: (1) The purpose of this study, whether there are risks and discomforts, whether it needs to be paid, whether it is completely voluntary, etc.; (2) The types of samples collected in this study and the details of the collection; (3) The personal information of the subjects, research-related measurement indicators, and physical examination information will be kept confidential. Informed consent will be voluntarily signed by individuals who agree to participate in this study. Basic information questionnaires, including body mass index, age, gender, and dietary habit etc., will be conducted through interviews. Blood and stool samples will be obtained as baseline samples from the participants before preoperative bowel preparation. During surgery, three samples from three different sites including tumor tissue, paracancerous tissue, and normal tissue will be collected in sterile tubes and stored in liquid nitrogen, then will be transferred to the $-80^{\circ} \mathrm{C}$ refrigerator until use. The participants will visit the hospital for postoperative chemotherapy about 3 weeks after surgery. Since the chemotherapy regimen will change with the cancer stage, only participants who receive XELOX chemotherapy (oxaliplatin + capecitabine) will continue to participate in this trial.

Participants in the prebiotic group ( $\mathrm{n}=25$ per group) will be asked to consume prebiotic (XOS, $3 \mathrm{~g} / \mathrm{day}$ ) along with routine capecitabine therapy. Participants in the control group $(n=50$ per group) will be asked to consume placebo (maltodextrin, $3 \mathrm{~g} /$ day). Prebiotics and placebo are white powder, packed in $3 \mathrm{~g} / \mathrm{bag}$. Since maltodextrin is colorless and tasteless after dissolution, it can reduce the chance of the placebo effect interfering with results. Each prebiotic bag (produced by Heagreen Company, Henan, China) contains $40.64 \%$ xylobiose, $27.75 \%$ xylotriose, $14.16 \%$ xylotetraose, $7.14 \%$ xylopentaose, $7.8 \%$ xylohexaose. Each box contains 30 bags. Participants will be instructed to mix one bag prebiotic or placebo with water and consume it every day. Participants will be given a box of prebiotics each time they visit the hospital for chemotherapy. The intervention will last for 12 weeks. Participants will be monitored for prebiotic consumption at each delivery of prebiotics and weekly telephone follow-up. Throughout the intervention, volunteers will be required to maintain their lifestyle, diet, as well as medications. They will be asked to collect stool and blood samples before ( 3 week) and after ( 15 week) the intervention and complete questionnaires in each chemotherapy cycle. Participants are asked to inform the investigators in time if there is any adverse event that may be associated with prebiotic. These adverse events will be recorded by investigators. Protocol modifications will be communicated to the ethics committee through an official modification request, and the participants will be notified by telephone.

\section{Data collection and sample handling}

The overall study design is depicted in Fig. 1. Assessment of participants will be conducted at the perioperative (baseline $=$ week 0), pre-treatment (week 3), post-treatment (week 15), and in each chemotherapy cycle. The primary outcomes will be the differences in the microbiota composition at different CRC tumor sites and differences in gut microbiota composition, adverse reactions, blood concentration in patients with CRC treated for 12 weeks with XOS or placebo. The secondary outcomes will include other blood indicators, SCFAs concentration, QoL, and mental health. 


\section{Gut microbial composition}

Tissue samples including tumor, para-carcinoma mucosa ( $2 \mathrm{~cm}$ away from the tumor), and normal mucosa (as far as possible from the tumor) will be obtained from LSCRC and RSCC participants during surgery. Each tissue will be cut into small pieces with a volume of 1 cubic centimeter and put into the cryopreserved tubes. After being frozen in liquid nitrogen, the tissue will be stored in the $-80{ }^{\circ} \mathrm{C}$ refrigerator for later use.

A total of $6 \mathrm{~g}$ stool samples from each participant will be collected in the morning for intestinal flora examination at baseline prior to preoperative bowel preparation and at 3 weeks (pre-treatment) and 15 weeks (post-treatment). Briefly, participants will be instructed to: (1) exhaust urine before placing disposable stool tray to prevent contamination of stool; (2) pass stool on the tray; (3) use a sterile spoon to dig up about $2 \mathrm{~g}$ stool from middle and posterior segment of stool and inserted into a sterilized screw cap containers marked with the participant's code and sampling date. For the purpose of reducing the change of microbiota composition in the stools, samples will be temporarily stored in a foam box with ice packs and transferred to the $-80^{\circ} \mathrm{C}$ refrigerator in the laboratory within two hours.

The total DNA of frozen samples will be extracted via QIAamp (QIAGEN) stool DNA kit according to manufacturer's instructions. DNA integrity and size will be assessed by the NanoDrop ND-1000 spectrophotometer (Thermo, USA) and 1\% agarose gel electrophoresis. DNA samples will be kept at $-80^{\circ} \mathrm{C}$ for later use. The bacterial $16 \mathrm{~S}$ rRNA V3-V4 regions will be amplified by polymerase chain reaction (PCR). High-throughput sequencing will be performed on an Illumina platform.

A sequence similarity threshold of $97 \%$ will be used to select operational taxonomic units (OTUs) and then perform taxonomy assignments via the Usearch platform (version $7.1 \mathrm{http}: / /$ drive5.com/uparse/). Principal co-ordinates analysis (PCOA) on weighted UniFrac distances will be measured on all OTUs by QIIME. Alpha diversity will be calculated through the Shannon index, Simpson index, and Chao1 metrics using mothur (version v.1.30.1 http://www.mothur.org/wiki/Schloss_SOP\#Alpha_diversity). Linear discriminant analysis (LDA) will be performed on the analysis software LEfSe (http://huttenhower.sph.harvard.edu/galaxy/). Subsequently, the communities or species that have significant differences in the sample division will be selected.

\section{Stool SCFA profiling}

Stool samples aliquots of $50 \mathrm{mg}$ g of dry weight each, will add with $50 \mu \mathrm{L}$ phosphoric acid, $100 \mu \mathrm{L}$ isohexanoic acid solution, and $400 \mu \mathrm{L}$ diethyl ether to homogenize. After centrifugation, the supernatant will be separated for gas chromatography-mass spectrometry (GC-MS) analysis. Stool SCFA profiling will be conducted using an Agilent Technology 6890 GC with autosamplers and 5973 MS Detection and Chemstation Data System (Agilent, Singapore). Chromatographic conditions will be as follows: Agilent HP-INNOWAX capillary column ( $30 \mathrm{~m} * 0.25 \mathrm{~mm} \mathrm{ID} * 0.25 \mu \mathrm{m})$; shunt injection, injection volume $1 \mu \mathrm{L}$, shunt ratio $10: 1$; injection port temperature of $250^{\circ} \mathrm{C}$; ion source temperature of $230^{\circ} \mathrm{C}$; initial oven temperature 
of $90^{\circ} \mathrm{C}, 10^{\circ} \mathrm{C} / \mathrm{min}$ up to $120^{\circ} \mathrm{C}, 5^{\circ} \mathrm{C} / \mathrm{min}$ up to $150^{\circ} \mathrm{C}, 25^{\circ} \mathrm{C} / \mathrm{min}$ up to $250^{\circ} \mathrm{C}$, and keep at $250^{\circ} \mathrm{C}$ for $2 \mathrm{~min}$. Helium will be used as gas carrier at a constant flow rate of $1 \mathrm{~mL} / \mathrm{min}$.

\section{Blood tests}

Venous blood samples will be collected before preoperative bowel preparation, at 3 weeks (pre-treatment) and 15 weeks (post-treatment). After fasting for 12 hours, venous blood will be collected into a coagulating tube in the morning and centrifuged at $3000 \mathrm{rpm}$ for 10 minutes for obtaining the upper serum (Thermo, USA). In addition to some of the usual blood biochemical indexes, we will use commercial enzyme-linked immunosorbent assay (ELISA) kits to assess the levels of inflammatory cytokines, including tumor necrosis factor- $a$ (TNF-a), interleukin-1 $\beta$ (IL-1 $\beta$ ), and interferon gamma (IFN- $\gamma$ ). Cellular immune indices, including T-lymphocytes (CD3+), helper inducer T cells (CD3+CD4+), suppressor/cytotoxic T cells (CD3+CD8+) will be measured by BD-FACS Canto II flow cytometer (Becton Dickinson, USA).

For the purpose of evaluating the bioavailability of chemotherapy drugs in participants, the blood concentration of capecitabine will be measured at 15 weeks (after intervention). On the third day of the fifth cycle of treatment (taking capecitabine for at least four meals), $5 \mathrm{~mL}$ venous blood samples will be collected 2 hours after taking medicine in the morning, put into a tube with ethylene diamine tetraacetic acid (EDTA) anticoagulant, and sent to the pharmacy department of the hospital for testing immediately.

\section{Adverse effects}

Adverse effects, including nausea, vomiting, diarrhea, leukopenia, anemia, thrombocytopenia, diarrhea, oral mucosal reaction, peripheral neuropathy, hand-foot syndrome, pigmentation, and abnormal liver function etc., will be assessed in each chemotherapy cycle using the Common Terminology Criteria for

Adverse Events (CTCAE) version 5.0 [40]. All adverse effects will be recorded from grades I to IV according to severity.

\section{Food records}

In order to minimize the interference of personal diet on the gut microbiota and record changes in dietary habits in time, volunteers will be asked to complete a 24-hour food record each time a stool sample is collected. They need to describe all foods and beverages consumed in detail including ingredients, weight, and cooking style. Daily nutrient intakes will be calculated based on the China Food Composition 2009 [41].

\section{Health-related QoL}


QoL will be assessed in each chemotherapy cycle using Chinese version of QoL questionnaire-caner 30 (QLQ-C30) formulated by the European Organization for Research and Treatment of Cancer. The questionnaire has 30 items, including 5 functional scales, 3 symptom scales, 6 individual measures items, and a global health [42].

\section{Mental health}

The prevalence of anxiety and depression in CRC patients is 13\%-57\%[43], and one of the important factors is the adverse effects of chemotherapy [44]. Hospital Anxiety and Depression Scale (HADS), an effective questionnaire with 14 items, will be used to assess depression and anxiety in each chemotherapy cycle. Each item is on a 4-point scale and final scores are proportional to the degree of anxiety and depression.

\section{Sample size calculation}

Since there is no clinical trial assessing the effect of intestinal flora on the bioavailability of chemotherapy drugs and the intervention endpoints of intestinal flora are currently unable to determine, the sample size is estimated based on the data from a previous clinical trial [22] which assessed the effects of synbiotics intervention on chemotherapy-related adverse effects (including diarrhea, appetite loss, nausea, and vomiting) for CRC patients received post-operative chemotherapy, showing that 20 volunteers per group had $90 \%$ power at an alpha level of 0.05 to detect significant differences.

Considering the long duration of the study (3 months) and the expected withdrawal of participants during the intervention, we plan to recruit $n=25$ participants per group $(n=100$ total).

\section{Data analysis}

The visiting time of the participants is in accordance with the treatment course, so as to facilitate the retention of the participants. The measured data for each visit will be collected on paper and then recorded electronically on a secure, locked office computer. The paper version of the data will be locked in a bookcase. Only the investigators running in the study will have access to the final study dataset.

The obtained data will be analyzed by SPSS software V26.0 (IBM, USA). Continuous data will be expressed as mean \pm standard deviation (SD), and categorical data will be expressed as the number of cases ( $n$ ) and percentage (\%). Differences in parameter continuous variables and asymmetric variables between groups will be analyzed through independent samples $t$ test and Mann-Whitney $U$ test, respectively. Using a paired $t$ test to assess the effect of the intervention in each group. The normality of data will be assessed using the Kolmogorov-Smirnov test.

Results will be regarded as statistically significant if $p$ values $<0.05$. 


\section{Discussion}

$\mathrm{CRC}$ is one of the most prevalent cancers world-wide. Postoperative adjuvant chemotherapy for CRC is a helpful attempt in recent years. However, the adverse effects during chemotherapy and the inter-individual variation in drug efficacy are still relatively big problems. Moreover, the different primary tumor locations may result in differences in intestinal flora composition and prognosis. Efforts to find a kind of safe food with therapeutic effect and to evaluate its impact on patients with CRC at different primary tumor locations are critical. Studies have revealed the vital part of intestinal flora in the progression and treatment of CRC $[3,12,45]$, and the concept of "pharmacomicrobiomics" is increasingly recognized [46]. Targeting intestinal flora, therefore, may modulate the efficacy and adverse effect of chemotherapy.

Both animal and human studies provide strong evidence that microecologics play essential roles in preventing chemotherapy-induced mucositis [47]. Bowen et.al. [48] have revealed the effects of VSL\#3, a mixture of Lactobacilli, bifidobacteria, and streptococcus, on preventing diarrhea following chemotherapy with irinotecan in rats. Synbiotics have significantly reduced the occurrence of severe lymphopenia and diarrhea, increased levels of Bifidobacterium and Lactobacillus species and concentrations of SCFAs in esophageal cancer patients undergoing neoadjuvant chemotherapy [49]. Dietary supplementation of prebiotics (oligofructose and inulin) can enhance the efficacy of six cytotoxic drugs and prolong the lifespan of rodents with transplantable malignant tumors [51,52]. XOS is a mixture of oligosaccharides which can be considered as a prebiotic[50]. XOS can modulate the diversity of intestinal flora, effectively multiply favorable bacteria such as bifidobacteria, and produce beneficial metabolites including SCFAs [53]. In addition, it has been reported that XOS are beneficial to type 2 diabetes mellitus [54], diarrhea [33], and constipation [32]. However, so far, there is no study evaluating the role of XOS consumption on the intestinal flora in patients with CRC.

This study will reveal the potential benefits of prebiotic for improving the gut microbiota composition, alleviating the adverse effects, and improving the efficacy of chemotherapy in patients with CRC. In addition, our study will provide data on the different distribution of tumor microbiota and the different changes of gut microbiota during treatment in LSCRC and RSCC, which may provide novel insights into personalized cancer treatment strategies based on primary tumor locations and gut microbiota in the future.

\section{Trial Status}

Recruitment began in June 2021 and is expected to be completed by May 2022.

\section{Abbreviations}

CRC: Colorectal cancer; SCFAs: Short-chain fatty acids; LSCC: Left-sided colorectal cancer; RSCC: Rightsided colon cancer; QoL: Quality of life; XOS: Xylooligosaccharide; SPIRIT: Standard Protocol Items: Recommendations for Interventional Trials; ULN: Upper limit of normal; PCR: Polymerase chain reaction; 
OTUs: Operational taxonomic units; PCoA: Principal co-ordinates analysis; LDA: Linear discriminant analysis; GC-MS: Gas chromatography-mass spectrometry; ELISA: Enzyme-linked immunosorbent assay; TNF-a: Tumor necrosis factor-a; IL-1 $\beta$ : Interleukin-1 $\beta$; IFN- $\gamma$ : Interferon gamma; CD3+: T-lymphocytes; CD3+CD4+: Helper inducer T cells; CD3+CD8+: Suppressor/cytotoxic T cells; EDTA: Ethylene diamine tetraacetic acid; CTCAE: Common Terminology Criteria for Adverse Events; QLQ-C30: QoL questionnairecaner 30; HADS: Hospital Anxiety and Depression Scale.

\section{Declarations}

\section{Ethics approval and consent to participate}

This study protocol (version 1, May 8, 2021) has been approved by an ethical committee of the Affiliated Hospital of Jiangnan University, Wuxi, China (LS2021026) and was registered at the Chinese Clinical Trial Registry (www.chictr.org.cn, ChiCTR2100046237). Informed consent will be voluntarily signed by individuals who agree to participate in this study.

\section{Consent for publication}

Not applicable.

\section{Competing interests}

The authors declare no competing interests.

\section{Funding}

The financial support is provided by Wuxi Project of Science and Technology Development (N20191001, N20192024) and Wuxi Translational Medicine Research Special Funding Project (ZZO03). The funders play no role in the study design or in the writing of this manuscript, nor did it affect data collection, analysis or manuscript publication.

\section{Availability of data and materials}

Not applicable.

\section{Authors' contributions}

All authors took part in the design of this study. YML was responsible for writing the entire manuscript, and $F Z$ and $W Z$ contributed to manuscript revising. $F Z$ was responsible for obtaining ethical approvals. $F Z$, WZ, and HC contributed to the acquisition of financial support. BJF, CQB, JMX, HXC, YM, XPC, and XG participated in the recruitment of participants. All authors have read and approved the final manuscript.

\section{Acknowledgements}

Not applicable. 


\section{Author details}

${ }^{1}$ State Key Laboratory of Food Science and Technology, School of Food Science and Technology, Jiangnan University, Wuxi 214122, China. ${ }^{2}$ Department of Nutrition, Affiliated Hospital of Jiangnan University, Wuxi 214122, China. ${ }^{3}$ Department of Gastrointestinal Surgery, Affiliated Hospital of Jiangnan University, Wuxi 214122, China. ${ }^{4}$ Department of Oncology, Affiliated Hospital of Jiangnan University, Wuxi 214122, China.

\section{References}

1. Sung H, Ferlay J, Siegel RL, Laversanne M, Soerjomataram I, Jemal A, Bray F. Global cancer statistics 2020: GLOBOCAN estimates of incidence and mortality worldwide for 36 cancers in 185 countries. CA-Cancer J Clin. 2021;71(3):209-49.

2. Kerr J, Anderson C, Lippman SM. Physical activity, sedentary behaviour, diet, and cancer: an update and emerging new evidence. Lancet Oncol. 2017;18(8):E457-71.

3. Bullman S, Pedamallu CS, Sicinska E, Claney TE, Zhang XY, Cai DN, Neuberg D, Huang K, Guevara F, Nelson T, et al. Analysis of Fusobacterium persistence and antibiotic response in colorectal cancer. Science. 2017;358(6369):1443-+.

4. Pleguezuelos-Manzano C, Puschhof J, Huber AR, van Hoeck A, Wood HM, Nomburg J, Gurjao C, Manders F, Dalmasso G, Stege PB, et al. Mutational signature in colorectal cancer caused by genotoxic pks(+)E. coli. Nature. 2020;580(7802):269-+.

5. Barton MK. Primary Tumor Location Found to Impact Prognosis and Response to Therapy in Patients With Metastatic Colorectal Cancer. CA-Cancer J Clin. 2017;67(4):259-60.

6. Siegel RL, Miller KD, Goding Sauer A, Fedewa SA, Butterly LF, Anderson JC, Cercek A, Smith RA, Jemal A. Colorectal cancer statistics, 2020. CA Cancer J Clin. 2020;70(3):145-64.

7. Tejpar S, Stintzing S, Ciardiello F, Tabernero J, Van Cutsem E, Beier F, Esser R, Lenz H-J, Heinemann V. Prognostic and Predictive Relevance of Primary Tumor Location in Patients With RAS Wild-Type Metastatic Colorectal Cancer Retrospective Analyses of the CRYSTAL and FIRE-3 Trials. JAMA oncology. 2017;3(2):194-201.

8. Petrelli F, Tomasello G, Borgonovo K, Ghidini M, Turati L, Dallera P, Passalacqua R, Sgroi G, Barni S. Prognostic Survival Associated With Left-Sided vs Right-Sided Colon Cancer A Systematic Review and Meta-analysis. JAMA oncology. 2017;3(2):211-9.

9. Loupakis F, Yang D, Yau L, Feng S, Cremolini C, Zhang W, Maus MKH, Antoniotti C, Langer C, Scherer SJ, et al. Primary Tumor Location as a Prognostic Factor in Metastatic Colorectal Cancer. JnciJournal of the National Cancer Institute 2015, 107(3).

10. Holch JW, Ricard I, Stintzing S, Modest DP, Heinemann V. The relevance of primary tumour location in patients with metastatic colorectal cancer: A meta-analysis of first-line clinical trials. Eur $\mathrm{J}$ Cancer. 2017;70:87-98. 
11. Arnold D, Lueza B, Douillard JY, Peeters M, Lenz HJ, Venook A, Heinemann V, Van Cutsem E, Pignon JP, Tabernero J, et al. Prognostic and predictive value of primary tumour side in patients with RAS wild-type metastatic colorectal cancer treated with chemotherapy and EGFR directed antibodies in six randomized trials. Ann Oncol. 2017;28(8):1713-29.

12. Rubinstein MR, Wang X, Liu W, Hao Y, Cai G, Han YW. Fusobacterium nucleatum promotes colorectal carcinogenesis by modulating E-cadherin/beta-catenin signaling via its FadA adhesin. Cell Host Microbe. 2013;14(2):195-206.

13. Donohoe DR, Holley D, Collins LB, Montgomery SA, Whitmore AC, Hillhouse A, Curry KP, Renner SW, Greenwalt A, Ryan EP, et al. A gnotobiotic mouse model demonstrates that dietary fiber protects against colorectal tumorigenesis in a microbiota- and butyrate-dependent manner. Cancer Discov. 2014;4(12):1387-97.

14. Stojanovska V, Sakkal S, Nurgali K. Platinum-based chemotherapy: gastrointestinal immunomodulation and enteric nervous system toxicity. American Journal of PhysiologyGastrointestinal Liver Physiology. 2015;308(4):G223-32.

15. McQuade JL, Daniel CR, Helmink BA, Wargo JA. Modulating the microbiome to improve therapeutic response in cancer. Lancet Oncol. 2019;20(2):E77-91.

16. Jia W, Li HK, Zhao LP, Nicholson JK. Gut microbiota: a potential new territory for drug targeting. Nat Rev Drug Discov. 2008;7(2):123-9.

17. Yu TC, Guo FF, Yu YN, Sun TT, Ma D, Han JX, Qian Y, Kryczek I, Sun DF, Nagarsheth N, et al. Fusobacterium nucleatum Promotes Chemoresistance to Colorectal Cancer by Modulating Autophagy. Cell. 2017;170(3):548-+.

18. Emorine M, Septier C, Andriot I, Martin C, Salles C, Thomas-Danguin T. The ellagic acid-derived gut microbiota metabolite, urolithin A, potentiates the anticancer effects of 5-fluorouracil chemotherapy on human colon cancer cells. Food Funct. 2015;6(5):1460-9.

19. Javdan B, Lopez JG, Chankhamjon P, Lee Y-CJ, Hull R, Wu Q, Wang X, Chatterjee S, Donia MS. Personalized Mapping of Drug Metabolism by the Human Gut Microbiome. Cell. 2020;181(7):1661-+.

20. Chang C-W, Liu C-Y, Lee H-C, Huang Y-H, Li L-H, Chiau J-SC, Wang T-E, Chu C-H, Shih S-C, Tsai T-H, et al. Lactobacillus casei Variety rhamnosus Probiotic Preventively Attenuates 5-

Fluorouracil/Oxaliplatin-Induced Intestinal Injury in a Syngeneic Colorectal Cancer Model. Frontiers in Microbiology 2018, 9.

21. Osterlund P, Ruotsalainen T, Korpela R, Saxelin M, Ollus A, Valta P, Kouri M, Elomaa I, Joensuu H. Lactobacillus supplementation for diarrhoea related to chemotherapy of colorectal cancer: a randomised study. Br J Cancer. 2007;97(8):1028-34.

22. He-Lei LU, Qin HL. Randomized controlled clinical study on synbiotics improvement of postoperative adverse reactions to chemotherapy in patients with colorectal cancer. Shanghai Journal of Preventive Medicine 2019, 31.

23. Chapla D, Pandit P, Shah A. Production of xylooligosaccharides from corncob xylan by fungal xylanase and their utilization by probiotics. Biores Technol. 2012;115(none):215-21. 
24. Yang H, Wang $K$, Song $X$, Feng $X$. Production of xylooligosaccharides by xylanase from Pichia stipitis based on xylan preparation from triploid Populas tomentosa. Biores Technol. 2011;102(14):7171-6.

25. Hald S, Schioldan AG, Moore ME, Dige A, Lærke HN, Agnholt J, Knudsen KEB, Hermansen K, Marco ML, Gregersen SJPO. Effects of Arabinoxylan and Resistant Starch on Intestinal Microbiota and Short-Chain Fatty Acids in Subjects with Metabolic Syndrome: A Randomised Crossover Study. 2016, 11(7):e0159223.

26. Ming-Hsu, Chen, Kelly S, Swanson GC, Fahey BS, Dien, Alison N, agricultural BJJo et al In Vitro Fermentation of Xylooligosaccharides Produced from Miscanthus $\times$ giganteus by Human Fecal Microbiota. 2016.

27. Nieto-Dominguez M, De Eugenio LI, York-Duran MJ, Rodriguez-Colinas B, Plou FJ, Chenoll E, Pardo E, Codoner F, Jesus Martinez MJFC. Prebiotic effect of xylooligosaccharides produced from birchwood xylan by a novel fungal GH11 xylanase. 2017, 232(OCT.1):105.

28. lida T, Yamada T, Hayashi N, Okuma K, Izumori K, Ishii R, Matsuo T. Reduction of abdominal fat accumulation in rats by 8-week ingestion of a newly developed sweetener made from high fructose corn syrup. Food Chem. 2013;138(2-3):781-5.

29. Yuanwen C, Yu Z, Chuan L, Dan D, Shaojun C. Research progress on physicochemical properties and applications of xylooligosaccharides. Food Industry 2019(No. 10):245-248.

30. Geraylou Z, Souffreau C, Rurangwa E, De Meester L, Courtin CM, Delcour JA, Buyse J, Ollevier FJF, Immunology S. Effects of dietary arabinoxylan-oligosaccharides (AXOS) and endogenous probiotics on the growth performance, non-specific immunity and gut microbiota of juvenile Siberian sturgeon (Acipenser baerii). 2013, 35(3):766-775.

31. Li Z, Summanen PH, Komoriya T, Sciences, SMFJIJoF, Nutrition. In vitro study of the prebiotic xylooligosaccharide (XOS) on the growth of Bifidobacterium spp and Lactobacillus spp. 2015, 66(8):919-922.

32. Tateyama I, Hashii K, Johno I, lino T, Hirai K, Suwa Y, Kiso Y. Effect of xylooligosaccharide intake on severe constipation in pregnant women. J Nutr Sci Vitaminol. 2005;51(6):445-8.

33. Carvalho A, Silva D, Pastore GM, Neto PJFRI. Xylo-oligosaccharides from lignocellulosic materials: Chemical structure, health benefits and production by chemical and enzymatic hydrolysis. 2013.

34. Mendis M, Simsek S. Arabinoxylans and human health. Food Hydrocolloids. 2014;42:239-43.

35. Volman JJ, Ramakers JD, Plat J. Dietary modulation of immune function by beta-glucans. Physiol Behav. 2008;94(2):276-84.

36. Gao Y, Zhang S, Li C, Xiao L, Shen J, Yin J. Acute and subchronic toxicity of xylo-oligosaccharide in mice and rats. Toxicol Mech Methods. 2012;22(8):605-10.

37. Oku T, Nakamura AS. Digestion, absorption, fermentation, and metabolism of functional sugar substitutes and their available energy. Pure Appl Chem. 2002;74(7):1253-61.

38. Yu X, Yin J, Li L, Luan C, Zhang J, Zhao C, Li S. Prebiotic Potential of Xylooligosaccharides Derived from Corn Cobs and Their In Vitro Antioxidant Activity When Combined with Lactobacillus. J Microbiol Biotechnol. 2015;25(7):1084-92. 
39. Chan A-W, Tetzlaff JM, Gotzsche PC, Altman DG, Mann H, Berlin JA, Dickersin K, Hrobjartsson A, Schulz KF, Parulekar WR, et al. SPIRIT 2013 explanation and elaboration: guidance for protocols of clinical trials. Bmj-British Medical Journal 2013, 346.

40. Freites-Martinez A, Santana N, Arias-Santiago S, Viera A. Using the Common Terminology Criteria for Adverse Events (CTCAE - Version 5.0) to Evaluate the Severity of Adverse Events of Anticancer Therapies. Actas Dermo-Sifiliograficas. 2021;112(1):90-2.

41. Yang Y, Pan GW,X. China food composition. 2 ed.: Beijing Medical Univ. Press; 2009.

42. Zhao H, Kanda K. Translation and validation of the Standard Chinese version of the EORTC QLQ-C30. Qual Life Res. 2000;9(2):129-37.

43. Walker J, Hansen $\mathrm{CH}$, Martin P, Sawhney A, Thekkumpurath P, Beale C, Symeonides S, Wall L, Murray G, Sharpe M. Prevalence of depression in adults with cancer: a systematic review. Ann Oncol. 2013;24(4):895-900.

44. Peng Y-N, Huang M-L, Kao C-H. Prevalence of Depression and Anxiety in Colorectal Cancer Patients: A Literature Review. International Journal of Environmental Research and Public Health 2019, 16(3).

45. Mima K, Nishihara R, Qian ZR, Cao Y, Sukawa Y, Nowak JA, Yang J, Dou R, Masugi Y, Song M, et al. Fusobacterium nucleatum in colorectal carcinoma tissue and patient prognosis. Gut. 2016;65(12):1973-80.

46. Alexander JL, Wilson ID, Teare J, Marchesi JR, Nicholson JK, Kinross JM. Gut microbiota modulation of chemotherapy efficacy and toxicity. Nature Reviews Gastroenterology Hepatology. 2017;14(6):356-65.

47. Redman MG, Ward EJ, Phillips RS. The efficacy and safety of probiotics in people with cancer: a systematic review. Ann Oncol. 2014;25(10):1919-29.

48. Bowen J, Stringer A, Gibson R, Yeoh A, Hannam S, Keefe D. VSL\#3 Probiotic treatment reduces chemotherapy-induced diarrhoea and weight loss. Cancer Biol Ther. 2007;6:1449-54.

49. Motoori M, Yano M, Miyata H, Sugimura K, Saito T, Omori T, Fujiwara Y, Miyoshi N, Akita H, Gotoh K, et al. Randomized study of the effect of synbiotics during neoadjuvant chemotherapy on adverse events in esophageal cancer patients. Clin Nutr. 2017;36(1):93-9.

50. Gibson GR, Roberfroid MB. DIETARY MODULATION OF, THE HUMAN COLONIC MICROBIOTA INTRODUCING THE CONCEPT OF PREBIOTICS. J Nutr. 1995;125(6):1401-12.

51. Taper HS, Roberfroid MB. Nontoxic potentiation of cancer chemotherapy by dietary oligofructose or inulin. Nutrition Cancer-an International Journal. 2000;38(1):1-5.

52. Taper HS, Roberfroid MB. Possible adjuvant cancer therapy by two prebioties - Inulin or oligofructose. In Vivo. 2005;19(1):201-4.

53. Aachary AA, Prapulla SG. Xylooligosaccharides (XOS) as an Emerging Prebiotic: Microbial Synthesis, Utilization, Structural Characterization, Bioactive Properties, and Applications. Comprehensive Reviews in Food Science Food Safety. 2011;10(1):2-16. 
54. Sheu WH-H, Lee IT, Chen W, Chan Y-C. Effects of Xylooligosaccharides in Type 2 Diabetes Mellitus. J Nutr Sci Vitaminol. 2008;54(5):396-401.

\section{Figures}

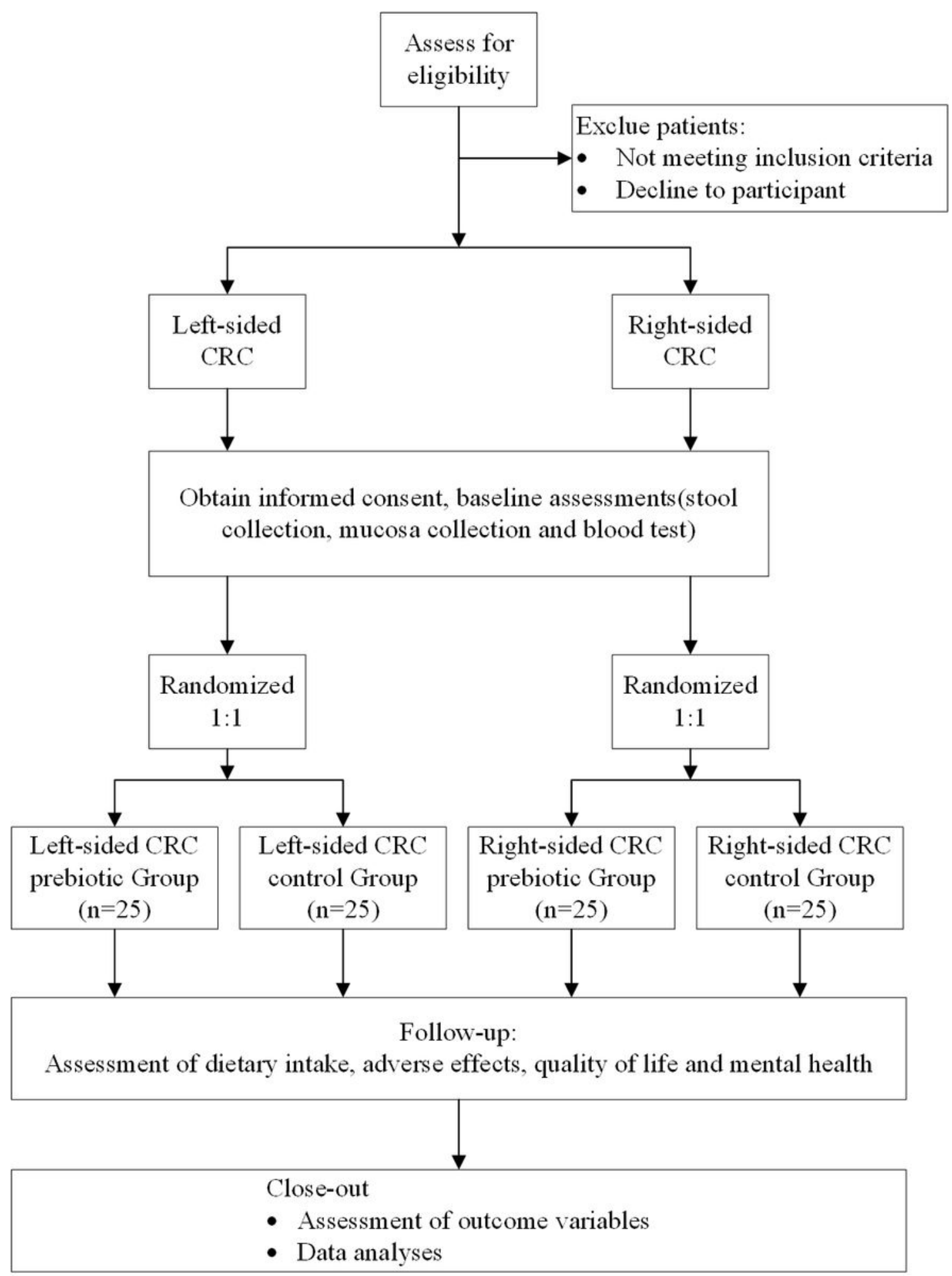

Figure 1 


\begin{tabular}{|c|c|c|c|c|c|c|c|}
\hline \multirow[b]{3}{*}{ TIMEPOINT } & \multicolumn{7}{|c|}{ STUDY'PERIOD } \\
\hline & \multirow{2}{*}{$\begin{array}{c}\text { Enrollment } \\
0\end{array}$} & \multirow{2}{*}{$\begin{array}{c}\text { Allocation } \\
\text { WK3 } \\
\text { Before } \\
\text { chemo }\end{array}$} & \multicolumn{4}{|c|}{ Post-allocation } & \multirow{2}{*}{$\begin{array}{c}\text { Close-out } \\
\text { WK15 } \\
\text { 5st cycle } \\
\text { chemo }\end{array}$} \\
\hline & & & $\begin{array}{c}\text { 1st' } \\
\text { cycle } \\
\text { chemo }\end{array}$ & $\begin{array}{c}2 \text { st } \\
\text { cycle } \\
\text { chemo }\end{array}$ & $\begin{array}{c}\text { 3st" } \\
\text { cycle } \\
\text { chemo }\end{array}$ & $\begin{array}{c}\text { 4st" } \\
\text { cycle } \\
\text { chemo }\end{array}$ & \\
\hline \multicolumn{8}{|l|}{ ENROLLMENT: } \\
\hline Eligibility screen & $\mathrm{X}$ & & & & & & \\
\hline Informed consent & $\mathrm{X}$ & & & & & & \\
\hline Allocation & & $\mathrm{X}$ & & & & & \\
\hline \multicolumn{8}{|l|}{ INTERVENTIONS: } \\
\hline \multicolumn{8}{|l|}{ Left-sided' prebiotic' group } \\
\hline Left-sided ' control' group & & & 4 & & & & \\
\hline \multicolumn{8}{|l|}{ Right-sided prebiotic group } \\
\hline Right-sided control group & & & 4 & & & & \\
\hline \multicolumn{8}{|l|}{ ASSESSMENTS: } \\
\hline Mucosa collection & $\mathrm{X}$ & & & & & & \\
\hline Stool collection & $\mathrm{X}$ & $\mathrm{X}$ & & & & & $\mathrm{X}$ \\
\hline SCFAs' concentration & & $\mathrm{X}$ & & & & & $\mathrm{X}$ \\
\hline Blood concentration & & & & & & & $\mathrm{X}$ \\
\hline Other" blood ' tests & $\mathrm{X}$ & $\mathrm{X}$ & & & & & $\mathrm{X}$ \\
\hline Adverse' effects & & & $\mathrm{x}$ & $\mathrm{x}$ & $\mathrm{X}$ & $\mathrm{X}$ & $\mathrm{X}$ \\
\hline Quality" of life & & $\mathrm{x}$ & $\mathrm{X}$ & $\mathrm{X}$ & $\mathrm{X}$ & $\mathrm{X}$ & $\mathrm{X}$ \\
\hline Mental'health & & $\mathrm{X}$ & $\mathrm{X}$ & $\mathrm{X}$ & $\mathrm{X}$ & $\mathrm{X}$ & $\mathrm{X}$ \\
\hline Food record & $\mathrm{X}$ & $\mathrm{X}$ & & & & & $\mathrm{X}$ \\
\hline
\end{tabular}

Figure 2

SPIRIT diagram: schedule of enrollment, interventions, and assessments

\section{Supplementary Files}


This is a list of supplementary files associated with this preprint. Click to download.

- SPIRITchecklist.docx 